O-band N-rich Silicon Nitride MZI based on GST

\title{
O-band N-rich Silicon Nitride MZI based on GST
}

\author{
Joaquin Faneca, ${ }^{1,2}$, a) Thalía Domínguez Bucio, ${ }^{2}$ Frederic $Y$. Gardes, ${ }^{2}$ and Anna Baldycheva ${ }^{1}$ \\ 1) Department of Engineering, University of Exeter, UK, N Park Rd, Exeter EX4 $4 Q F$ \\ ${ }^{2)}$ Optoelectronics Research Centre, B53, University of Southampton, SO17 1BJ, UK \\ (Dated: 18 February 2020)
}

We have experimentally demonstrated an O-band Mach-Zehnder interferometer (MZI) based on a N-rich silicon nitride platform combined with $\mathrm{Ge}_{2} \mathrm{Sb}_{2} \mathrm{Te}_{5}$ for future optical communication applications. The device operation relies on controlling the waveguide's losses using a phase change material cell which can be changed from amorphous (lowloss) to crystalline (high-loss). An extinction ratio (ER) as high as $11 \mathrm{~dB}$ was obtained between the amorphous (ON) and the crystalline (OFF) states of the MZI optical building block. The insertion loss of the MZI structure per cell unit length was measured to be as high as $0.87 \mathrm{~dB} / \mu \mathrm{m}$ in OFF state and as low as $0.064 \mathrm{~dB} / \mu \mathrm{m}$ in ON state for TM polarisation.

Silicon Photonics is a technology that has emerged to provide CMOS based low-cost fabrication of photonic integrated circuits (PICs) for high speed optical data manipulation and transmission. ${ }^{1,2}$ Both start-up and established companies are actively developing CMOS based photonics as the future technology for high speed data interconnects and high performance computing. ${ }^{3}$ Amongst the CMOS enabling materials, silicon nitride ( $\mathrm{SiNx}$ ) provides a promising and complementary platform for the development of low cost CMOS compatible waveguides and related photonic components. This is due to its low-temperature fabrication, the ability to precisely tailor its optical properties, and the high thermal stability of the final devices. ${ }^{4,5}$ Although $\mathrm{Si}$ waveguides provide a tighter confinement that leads to more compact devices, $\mathrm{SiNx}$ as a deposited material in their stoichiometric and non-stoichiometric forms is more cost effective and provides more freedom in the construction of more complex multilayer photonics circuitry. As a result, SiNx waveguides have been widely employed for light propagation in the near infrared and the visible range of the electromagnetic spectrum. ${ }^{6,7}$

To date, one of the main challenges faced by silicon photonics is the lack of volatile and non volatile reconfigurable components, ${ }^{8}$ which prevents the development of devices combining both functionalities such as switches, filters, an memories. ${ }^{9,10}$ One of the main emerging material families that can provide an alternative to non volatile circuit reconfigurability comprises phase change materials (PCMs).${ }^{11-13}$ PCMs have two (or more) reversible states that exhibit large changes in their optical properties ${ }^{14,15}$ which make them the most promising candidates to address non volatile reconfig urability in CMOS photonic devices. ${ }^{16-18}$ The phase change process can be thermally, optically or electrically driven. The modulation speed depends on the switching mechanism and device geometry. Optical switching in integrated photonic devices reaches "ns" modulation $(\sim \mathrm{MHz}),{ }^{12}$ but faster modulation rates have been demonstrated in the sub-ns regime $(\sim \mathrm{GHz})^{19}$ by electrical switching, using carbon nanotube electrodes. ${ }^{20}$ Using micro-heaters, switching times conventionally lays in the order of " $\mu \mathrm{s}$ ", leading to lower modulation rates $(\sim \mathrm{kHz}) \cdot{ }^{21}$ Crystallization is achieved by heating

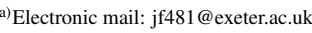

the material above the glass transition temperature and then slowly cooling it down, leading to nucleation followed by the growth of the nuclei over a small distance until they impinge on other crystallites. Returning to the amorphous state is more challenging, and requires the PCM to be molten and then very rapidly quenched..$^{22}$

The combination of SiNx photonic waveguides and PCMs can lead to fast (ns) non volatile reconfigurable technologies for optical communications applications. ${ }^{23,24}$ Different silicon and SiNx building blocks have been explored in the Cband using PCMs as the reconfigurable material. ${ }^{25,26}$ In this work, we present an O-band N-Rich Silicon Nitride MachZehnder Interferometer (MZI) based on the commonly used $\mathrm{Ge}_{2} \mathrm{Sb}_{2} \mathrm{Te}_{5}$ (GST) phase change material, providing a nonvolatile material for applications in the O-band for optical communications (Fig. 1).

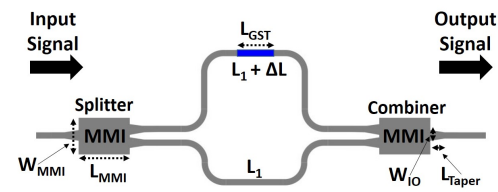

FIG. 1. Schematic of the MZI with a GST patch of the length $\mathrm{L}_{\mathrm{GST}}=$ 5,10 and $15 \mu \mathrm{m}$ deposited on the longer arm (top arm) of the length $\mathrm{L}_{1}+\Delta \mathrm{L}$, where $\mathrm{L} 1=614 \mu \mathrm{m}$ and $\Delta \mathrm{L}=20,40$ and $60 \mu \mathrm{m}$. MMI structure with width $\mathrm{W}_{\mathrm{MMI}}=15 \mu \mathrm{m}$ and length $\mathrm{L}_{\mathrm{MMI}}=168 \mu \mathrm{m}$ are used as splitters/combiners. The width of the single mode waveguide $(700 \mathrm{~nm})$ is tapered to $\mathrm{W}_{\mathrm{IO}}=6 \mu \mathrm{m}$ with tapers of length $\mathrm{L}_{\text {taper }}=100$ $\mu \mathrm{m}$ to increase the fidelity of the MMIs.

The optical platform consists of a $700 \mathrm{~nm}$ wide SiNx strip waveguide formed by etching a $600 \mathrm{~nm}$ thick SiNx layer. ${ }^{27}$ This design allows both single TE and TM mode propagation with low losses in the O-band $(<1 \mathrm{~dB} / \mathrm{cm})$. The propagation losses measured with this geometry account for both the material absorption losses and the scattering losses produced due to the sidewall roughness of the waveguides. The asymmetric MZI structure is built with two multi-mode interferometers (MMI) that split the input signal into two arms of different length and then combine them into an output signal. A thin layer $(15 \mathrm{~nm})$ of phase-change material $(\mathrm{PCM})$ is deposited 
on top of the longer arm of the asymmetric MZI with cells of different length $(5,10$ and $15 \mu \mathrm{m})$, with a $10 \mathrm{~nm}$ thick protective $\mathrm{SiO}_{2}$ encapsulation layer. By using an asymmetric MZI design, we enable engineering of the device performance in two ways: by tuning the length difference between the arms $(\Delta \mathrm{L})$ and the geometry of the deposited GST cell.

A PCM cell thickness of $15 \mathrm{~nm}$ was selected as it provides a good balance between attenuation and total transmission in the device between states (Fig. 2). In this work we focused on the phase-change alloy GST, due to the well-established fabrication methodology and most importantly, due to thorough characterisation of optical properties in both phases, which determine the partial attenuation of the guided modes, along with the characteristic non-volatile tunability and switching times within the nanosecond scale. ${ }^{28,29}$ This reconfigurable material provides a suitable platform for stable optical modulation exploiting Mach-Zehnder interferometers in the Oband, as demonstrated in this work. In order to estimate the waveguide transmissivity, losses, and the interferometer performances in terms of modulation and frequency selectivity, we numerically analysed the mode profile of each interferometer branch via COMSOL Multiphysics. TM polarization was selected due to the grating couplers fabrication optimization and the stronger mode overlap compared with the TE mode (see supplementary material, Figure S1). The straight waveguide without GST layer demonstrated lossless propagation with real effective refractive index value for TM mode of $n_{\text {eff }}=1.6518$. For the hybrid waveguide with the PCM cell, which represents the longer arm of the assymetrical MZI, the phase of the GST layer and its effect on the different refractive index of the thin layer (Fig. 2(a-b)) was studied for both amorphous and crystalline states. For each case scenario, we obtain $n_{e f f}$. The real part of the effective refractive index $\left(n_{e f f}^{r}\right)$ is related with the propagation constant and the imaginary part $\left(n_{e f f}^{i}\right)$ is related with the absorption due to the phase state of the phase change material (amorphous or crystalline). The optical constants used in the simulations at $1310 \mathrm{~nm}$ are $n_{S i N x}=1.92, n_{G S T}^{a}=3.96856+j 0.23$, $n_{G S T}^{c}=6.15+j 1.57, n_{S_{i O} O_{2}}=1.4468$ and $n_{P M M A}=1.4823$. This resulted in values for the TM mode effective refractive index of $n_{e f f}^{a}=1.67+j 1.8 \cdot 10^{-3}$ and $n_{e f f}^{c}=1.68+j 0.016$ for the amorphous and crystalline states respectively (Fig. 2(ab)). An interface loss due to the reflection between the bare (no GST) and the hybrid (GST) structure in the longer arm of the MZI waveguide is $0.11 \mathrm{~dB}$ for the crystalline state and is $0.03 \mathrm{~dB}$ for the amorphous state. Consequently, the difference in the interface losses due to reflection between the amorphous and crystalline states is as low as $0.08 \mathrm{~dB}$ per interface (see supplementary material, Figure S2). The theoretica values we have used for the simulations of the crystalline and amorphous state have been measured using ellipsometry and are consistent with published values in Ref. ${ }^{30,31}$ The absorption and the phase shift of the phase change material strongly depend on the thickness of the PCM as shown in (Fig. 2(c-d)) for the amorphous and crystalline states.

The assymetric MZIs were fabricated on a $600 \mathrm{~nm}$ thick nitrogen rich $\mathrm{SiN}_{\mathrm{x}}$ layer with a refractive index of 1.92 at a wavelength of $1310 \mathrm{~nm}$. This layer was deposited on a 8 " $(200$
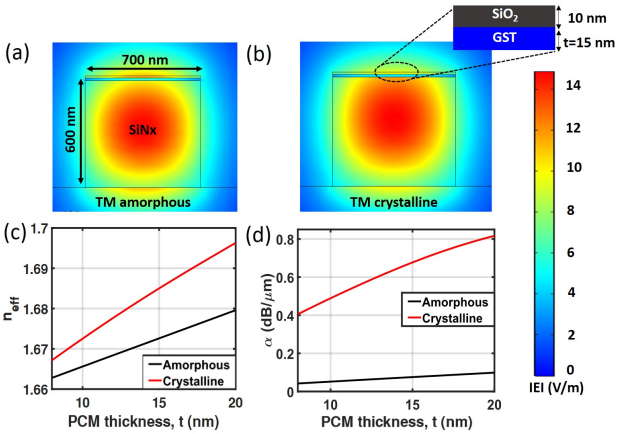

FIG. 2. Eigenmode simulation of fundamental TM optical mode propagating with GST layer on top in the (a) amorphous state, using PMMA as cladding and (b) crystalline, using $\mathrm{SiO}_{2}$ as cladding; (c) effective refractive index and (d) mode attenuation both as a function of cell thickness.

mm) $\mathrm{Si}$ wafer with a $2 \mu \mathrm{m}$ thick thermally grown $\mathrm{SiO}_{2}$ layer using a NH3-free plasma enhanced chemical vapour deposition (PECVD) process detailed in $\operatorname{Ref}^{4}$ and Ref. ${ }^{27}$ The device layout was defined on the wafer using deep ultraviolet (DUV) lithography using a wavelength of $248 \mathrm{~nm}$. The written design was then transferred to the SiNx layer using standard inductively coupled plasma (ICP) etching with an etch depth of $600 \mathrm{~nm}$ and a $\mathrm{SF}_{6}: \mathrm{CHF}_{3}$ chemistry. The GST cells capped by $10 \mathrm{~nm}$ of amorphous $\mathrm{SiO}_{2}$ were then integrated on top of the MZIs using physical vapour deposition (PVD) of GST thin film through a lithography mask (see supplementary material, Figure S3).

The Mach-Zehnder interferometer, was fabricated with a difference between the arm length $(\Delta L)$ of 40,60 and $80 \mu \mathrm{m}$. Three different GST cells with thickness of $15 \mathrm{~nm}$, lengths of 5,10 and $15 \mu \mathrm{m}$, and $700 \mathrm{~nm}$ width were deposited on top of the longer arm of the MZIs, capped by a second layer of $10 \mathrm{~nm}$ amorphous $\mathrm{SiO}_{2}$ to prevent oxidation. When the fabrication was completed, the devices were spin coated with Polymethyl methacrylate (PMMA) to enhance performance (optimize insertion loss of the grating couplers). PMMA was selected due to its similar optical properties to $\mathrm{SiO}_{2}$ at 1310 $\mathrm{nm}$, and room temperature process, avoiding thermal crystallization of the GST. Once the amorphous state was characterized, PMMA was removed and we induced the crystallization of the phase change material during the silicon oxide PCVD deposition process when temperatures as high as $350{ }^{\circ} \mathrm{C}$ are reached ${ }^{32}$ changing the PCM to its crystalline state.

The phase transition is thermally driven (in this case the CVD chamber acted as an oven), GST forms a metastable distorted rocksalt phase, ${ }^{33}$ in which Te atoms occupy one sublattice, while $\mathrm{Ge}, \mathrm{Sb}$, and vacancies occupy the second one in a random fashion. With sufficient thermal energy crystallites will form within the amorphous matrix (nucleation), and grow. ${ }^{34}$ SEM and optical microscopy images were taken for 
the different asymmetric MZI structures with the GST cell deposited on the longer arm (Fig. 3)
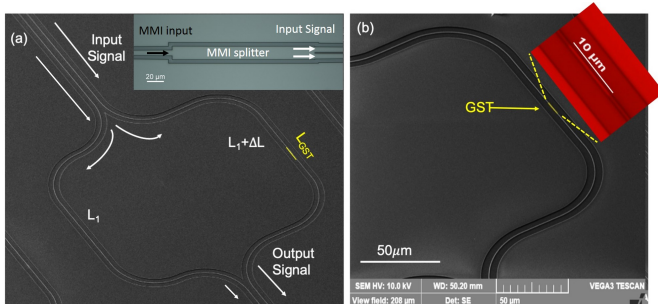

FIG. 3. (a) SEM image of an asymmetric MZI based on SiNx and phase change material (GST), SEM image of the MMI splitter as an inset. (b) Zoom in image verifying the position of the phase change material cell (yellow) deposited on the longer arm of the MZI.

Using the constructive interference equation of a MZI, the experimental effective refractive index of the waveguide can be determined as:

$$
m \cdot \lambda_{m}=n_{e f f} \Delta L
$$

where $\lambda_{m}$ is the resonant wavelength, $m$ is the filter order $n_{\text {eff }}$ the effective refractive index of the bare $700 \times 600 \mathrm{~nm}$ ridge waveguide mode coated with PMMA on top, and $\Delta L$ is the difference between the length of the two arms of the MZI We fabricated a bare MZI with $\Delta L=20 \mu \mathrm{m}$ and filter order of $m=25$. Using the spectral response of the fabricated MZI (Fig. 4), the TM effective refractive index of the $700 \times 600$ ridge waveguide structure can be obtained $\left(n_{e f f}=1.63\right)$. A free spectral range (FSR) of $46 \mathrm{~nm}$ and ER of $22.73 \mathrm{~dB}$ have been measured.

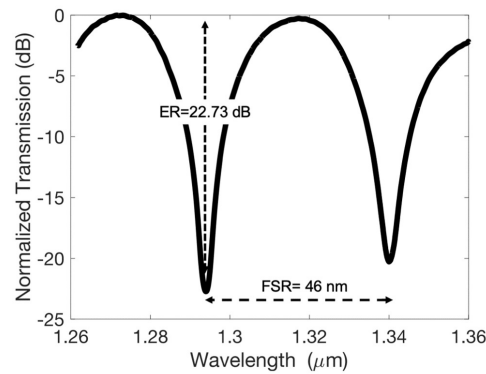

FIG. 4. Bare MZI interferometer spectrum for a difference between the arms of $\Delta L=20 \mu \mathrm{m}$

The spectral response of the MZIs for different cell lengths for both amorphous and crystalline states of the phase change material were characterised using an Agilent 8164B tuneable laser source with a tunable wavelength of 1260 to $1320 \mathrm{~nm}$ (Fig 5). The polarisation of the light was controlled to ensure that only TM modes could propagate through the devices. All MZIs structures were connected to input and output grating couplers consisting of a $10 \mu \mathrm{m}$ width and $40 \mu \mathrm{m}$ length with a period of $950 \mathrm{~nm}$ tapered down to a single-mode waveguide of $700 \mathrm{~nm}$. The angle of the optical fibres was selected to be $14^{\circ}$ to ensure maximum coupling at the wavelength of interest $(1310 \mathrm{~nm})$.

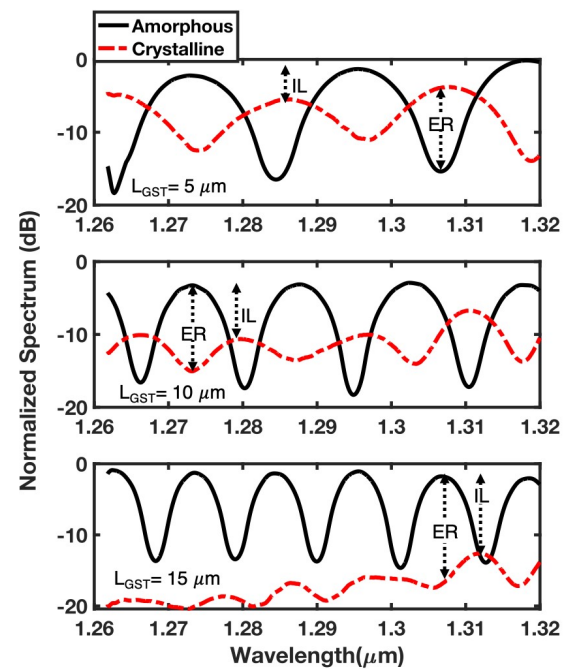

FIG. 5. Experimental normalized transmission from top to bottom of a MZI with $\Delta L=40 \mu \mathrm{m}$ and $L_{G S T}=5 \mu \mathrm{m}$ in the amorphous state (black) and in the crystalline state (red) [Top Figure]. Experimental normalized transmission for a MZI with $\Delta L=60 \mu \mathrm{m}$ and $L_{G S T}=10$ $\mu \mathrm{m}$ in the amorphous state (black) and in the crystalline state (red) [Mid Figure]. Experimental normalized transmission for a MZI with $\Delta L=80 \mu \mathrm{m}$ and $L_{G S T}=15 \mu \mathrm{m}$ in the amorphous state (black) and in the crystalline state (red) [Bottom Figure].

By switching the state of the GST cell, an optical switch is demonstrated in the O-band. The normalized transmission was calculated by removing the grating couplers contribution to optical insertion losses. Consequently, the measured optical losses are intrinsic to the MZI device. Values for the MZI device losses (DLs) of $0.14 \pm 0.02,0.42 \pm 0.03$ and $0.98 \pm 0.04$ $\mathrm{dB}$ are measured in the amorphous state of the phase change material for 5, 10 and $15 \mu \mathrm{m}$ cell, respectively. For the crystalline state, MZI optical insertion loss values are measured as high as $3.9 \pm 0.8 \mathrm{~dB}, 7.1 \pm 1.7 \mathrm{~dB}$ and $13.4 \pm 1.6 \mathrm{~dB}$, for the different cell lenghts $(5,10$ and $15 \mu \mathrm{m})$. The theoretical MZI optical losses for the amorphous and crystalline states of the cell are $0.075 \mathrm{~dB} / \mu \mathrm{m}$ and $0.68 \mathrm{~dB} / \mu \mathrm{m}$ respectively. The experimental losses measured in this device are $0.064 \mathrm{~dB} / \mu \mathrm{m}$ (ON-state) and $0.87 \mathrm{~dB} / \mu \mathrm{m}$ (OFF-state) (Fig 6). The discrepancy between experimental and theoretical results are due to the thickness of the GST cell and fabrication defects that affect the effective refractive index calculation. ${ }^{18}$ The difference 
in the insertion loss between the amorphous and crystalline states is the key component for future non-volatile reconfigurable switches in the O-band. An extinction ratio (ER) as high as $11.5 \mathrm{~dB}$ in transmission was measured for a $L_{G S T}=$ $5 \mu \mathrm{m}$ at $1307 \mathrm{~nm}$ and $\Delta L=40 \mu \mathrm{m}$ with $4 \mathrm{~dB}$ insertion loss (IL). IL was calculated using the difference between the DL of the two different material states. Increasing the length of the cell to $L_{G S T}=10 \mu \mathrm{m}$ and $\Delta L=60 \mu \mathrm{m}$ results in an ER of $12.3 \mathrm{~dB}$ at $1273 \mathrm{~nm}$ with an insertion loss of $7 \mathrm{~dB}$. Finally, for a cell of $L_{G S T}=15 \mu \mathrm{m}$ and $\Delta L=80 \mu \mathrm{m}$, a $13 \mathrm{~dB}$ extinction ratio was measured at $1310 \mathrm{~nm}$, with an insertion loss of $12 \mathrm{~dB}$ (Fig. 6). We have experimentally demonstrated an extinction ratio (ER) difference between the amorphous and crystalline states for the different cells lengths greater than $11 \mathrm{~dB}$. The optical switch due to the change of the phase change material state (amorphous or crystalline) allows us to distinguish between different transmission levels. Using the GST based MZI demonstrated in this paper, future non-volatile memories and switches with the improved ER, compared with a straight waveguide design, can be fabricated and further explored in the O-band.

Different technologies such as: liquid crystals, ${ }^{35}$ graphene, ${ }^{36,37}$ p-n junctions, ${ }^{38}$ and micro-heaters ${ }^{39}$ have been used as basic components to create silicon or silicon nitride based MZI switches. Other platforms such as $\mathrm{LiNbO}_{3}{ }^{40,41}$ or electro-absorption ${ }^{42,43}$ based switches have been demonstrated and they present an alternative to the CMOS compatible silicon photonic platform. All these technologies are volatile with switching mechanism being thermo-optic (T-O) or electro-optic (E-O), while the change in the refractive index between states is relatively small $(\Delta \mathrm{n} \sim 0.21)$. Using non-volatile phase change materials, specifically GST, the refractive index difference can be achieved as high as $\Delta \mathrm{n} \sim 2$, however, the losses of the device in the crystalline state are also increased in comparison to the other technologies. An overview table which compares different MZI switches technologies is presented in Table. I.

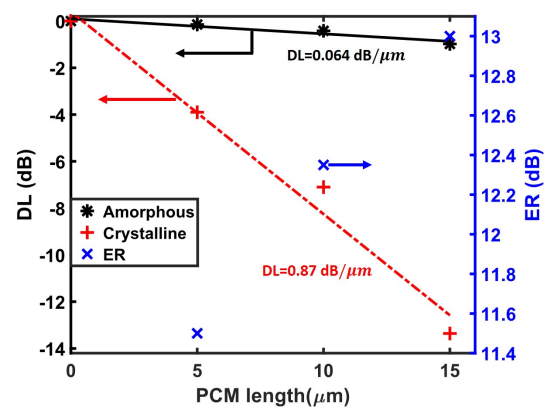

FIG. 6. Device Losses (DLs) of the MZI device for the amorphous state (black markers) and crystalline state (red markers) with a linear fitting for the amorphous state (black line) and for the crystalline state (red line) for different GST cell lengths (left axis), Extinction ratio (ER) of the MZI for the different length of the cell (right axis)
TABLE I. Comparison between this work ${ }^{*}$ and the state-of-the-art of MZI based switches. ${ }^{a}$ Estimated value.

\begin{tabular}{|c|c|c|c|c|c|c|}
\hline & $\begin{array}{c}\text { IL } \\
(\mathrm{dB})\end{array}$ & $\begin{array}{c}\text { ER } \\
\text { (dB) }\end{array}$ & $\begin{array}{c}\text { Switching } \\
\text { time }\end{array}$ & $\begin{array}{c}\text { Power } \\
\text { (W) }\end{array}$ & $\begin{array}{c}\text { Footprint } \\
\left(\mathrm{mm}^{2}\right)\end{array}$ & Technology \\
\hline MZI - GST* & $7-12$ & $11-13$ & $\mathrm{~ns}^{\mathrm{a}}$ & $0.1^{\mathrm{a}}$ & 0.4 & Non-volatile \\
\hline T-O MZI ${ }^{39,44-46}$ & $4-22$ & $15-35$ & $30-250 \mu \mathrm{s}$ & $0.07-1.9$ & $0.12-2.62$ & Volatile \\
\hline E-O MZI ${ }^{47-50}$ & $6-17$ & $10-30$ & ps & $0.05-1.2$ & $0.04-0.05$ & Volatile \\
\hline
\end{tabular}

We have demonstrated, theoretically and experimentally, an O-band SiNx MZI based on phase change materials for optical communications applications. The difference in the attenuation between the amorphous and crystalline state can provide the silicon photonics industry the opportunity of fabrication and generation of optical switches and attenuators in this range of the spectrum. A TM insertion loss as high as $0.87 \mathrm{~dB} / \mu \mathrm{m}$ was measured for the MZI structure per cell unit length in OFF state and $0.064 \mathrm{~dB} / \mu \mathrm{m}$ in ON state. We have demonstrated an experimental improvement in the E.R up to 11,12 and $13 \mathrm{~dB}$ compared with what is typical of a straight waveguide $2.5,5$ and $7.5 \mathrm{~dB}$ for all the different cell lengths 5,10 and $15 \mu \mathrm{m}$ between the amorphous and crystalline states respectively. This work provides scope to open the versatile non-volatile reconfigurable components in the O-band and provide solutions for non volatile circuit and reconfigurability for photonic integrated circuits.

See the supplementary material for detailed mode analysis. The model to calculate the difference in the interface losses due to reflection between the amorphous and crystalline states, and finally, fabrication process flow can also be found in the supplementary material.

We acknowledge financial support from: The Engineering and Physical Sciences Research Council (EPSRC) of the United Kingdom via the EPSRC (Grant No. EP/L015331/1, Grant No. EP/N035569/1, EP/N013247/1, EP/L021129/1 and $\mathrm{EP} / \mathrm{R} 003076 / 1)$ and the EU project id 688516 COSMICC

\section{REFERENCES}

G. T. Reed, G. Mashanovich, F. Y. Gardes, and D. J. Thomson, "Silicon optical modulators," Nature Photonics 4, 518-526 (2010).

${ }^{2}$ N. Sherwood-Droz, H. Wang, L. Chen, B. G. Lee, A. Biberman, K. Bergman, and M. Lipson, "Optical $4 \times 4$ hitless Silicon router for optical Networks-on-Chip (NoC): erratum." Optics express 16, 19395 (2008). ${ }^{3}$ D. Thomson, A. Zilkie, J. E. Bowers, T. Komljenovic, G. T. Reed, L. Vivien, D. Marris-Morini, E. Cassan, L. Virot, J.-M. Fédéli, J.-M. Hartmann, J. H. Schmid, D.X. Xu, F. Boeuf, P. O'Brien, G. Z. Mashanovich,

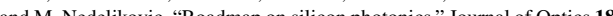
and M. Nedeljkovic, 'Roadmap on silicon photonics,' Journal of Optics 18 073003 (2016)

T. Domínguez Bucio, A. Z. Khokhar, G. Z. Mashanovich, and F. Y. Gardes, "Athermal silicon nitride angled mmi wavelength division (de)multiplexers for the near-infrared," Opt. Express 25, 27310-27320 (2017).

${ }^{5}$ T. Domínguez Bucio, A. Z. Khokhar, C. Lacava, S. Stankovic, G. Z. Mashanovich, P. Petropoulos, and F. Y. Gardes, "Material and optical properties of low-temperature NH3-free PECVD SiNx layers for photonic applications," Journal of Physics D: Applied Physics 50, 025106 (2016).

${ }^{6}$ J. N. Milgram, J. Wojcik, P. Mascher, and a. P. Knights, "Optically pumped Si nanocrystal emitter integrated with low loss silicon nitride waveguides." Optics express 15, 14679-14688 (2007). 
${ }^{7}$ S. Gaugiran, S. Gétin, J. Fedeli, G. Colas, A. Fuchs, F. Chatelain, and J. Dérouard, "Optical manipulation of microparticles and cells on silicon nitride waveguides." Optics express 13, 6956-6963 (2005).

${ }^{8}$ R. Soref, "Tutorial: Integrated-photonic switching structures," APL Photonics 3, 021101 (2018).

${ }^{9}$ J. Faneca, T. S. Perova, V. Tolmachev, and A. Baldycheva, "Onedimensional multi-channel photonic crystal resonators based on silicon-oninsulator with high quality factor," Frontiers in Physics 6, 33 (2018).

${ }^{10}$ J. Faneca, B. T. Hogan, I. R. Diez, F. Y. Gardes, and A. Baldycheva, "Tuning silicon-rich nitride microring resonances with graphene capacitor for high-performance computing applications," Optics Express 27, 3512935140 (2019).

${ }^{11}$ C. Ríos, M. Stegmaier, P. Hosseini, D. Wang, T. Scherer, C. D. Wright,

H. Bhaskaran, and W. H. P. Pernice, "Integrated all-photonic non-volatile multi-level memory," Nature Photonics 9, 725-732 (2015).

${ }^{12}$ C. Rios, P. Hosseini, C. D. Wright, H. Bhaskaran, and W. H. P. Pernice, "On-chip photonic memory elements employing phase-change materials," Advanced Materials 26, 1372-1377 (2014).

${ }^{13}$ C. Rios, M. Stegmaier, Z. Cheng, N. Youngblood, C. D. Wright, W. H. P. Pernice, and H. Bhaskaran, "Controlled switching of phase-change materials by evanescent-field coupling in integrated photonics [Invited]," Optical Materials Express 8, 2455 (2018).

${ }^{14}$ W. Wełnic, A. Pamungkas, R. Detemple, C. Steimer, S. Blügel, and M. Wuttig, "Unravelling the interplay of local structure and physical properties in phase-change materials," Nature Materials 5, 56-62 (2006).

${ }^{15} \mathrm{~W}$. Wełnic, S. Botti, L. Reining, and M. Wuttig, "Origin of the optical contrast in phase-change materials," Physical Review Letters 98, 1-4 (2007).

${ }^{16} \mathrm{M}$. Wuttig, H. Bhaskaran, and T. Taubner, "Phase-change materials for non-volatile photonic applications," Nature Photonics 11, 465-476 (2017).

${ }^{17} \mathrm{M}$. Wuttig and N. Yamada, "Phase-change materials for rewriteable data storage," Nature Materials 6, 824 (2007)

${ }^{18}$ H. Zhang, L. Zhou, B. M. A. Rahman, X. Wu, L. Lu, Y. Xu, J. Xu, J. Song, Z. Hu, L. Xu, and J. Chen, "Ultracompact si-gst hybrid waveguides for nonvolatile light wave manipulation," IEEE Photonics Journal 10, 1-10 (2018).

${ }^{19}$ N. Ciocchini, M. Laudato, M. Boniardi, E. Varesi, P. Fantini, A. L. Lacaita, and D. Ielmini, "Bipolar switching in chalcogenide phase change memory," Scientific reports 6, 29162 (2016).

${ }^{20}$ F. Xiong, A. D. Liao, D. Estrada, and E. Pop, "Low-power switching of phase-change materials with carbon nanotube electrodes," Science 332, 568-570 (2011)

${ }^{21}$ J. Zheng, Z. Fang, C. Wu, S. Zhu, P. Xu, J. K. Doylend, S. Deshmukh, E. Pop, S. Dunham, M. Li, et al., "Nonvolatile electrically reconfigurable integrated photonic switch," arXiv preprint arXiv:1912.07680 (2019)

${ }^{22}$ Z. Cheng, C. Ríos, W. H. P. Pernice, C. D. Wright, and H. Bhaskaran, "On-chip photonic synapse _ Supplementary Material," Science Advance 1700160, 1-7 (2017)

${ }^{23}$ J. Zheng, A. Khanolkar, P. Xu, S. Colburn, S. Deshmukh, J. Myers, J. Frantz, E. Pop, J. Hendrickson, J. Doylend, N. Boechler, and A. Majumdar, "Gst-on-silicon hybrid nanophotonic integrated circuits: a nonvolatile quasi-continuously reprogrammable platform," Opt. Mater. Express 8, 1551-1561 (2018)

${ }^{24}$ K. J. Miller, R. F. Haglund, and S. M. Weiss, "Optical phase change materials in integrated silicon photonic devices: review," Opt. Mater. Express 8 , 2415-2429 (2018)

${ }^{25}$ A. Sebastian, M. Le Gallo, G. W. Burr, S. Kim, M. BrightSky, and E. Eleftheriou, "Tutorial: Brain-inspired computing using phase-change memory devices," Journal of Applied Physics 124, 111101 (2018).

${ }^{26}$ E. Gemo, S. G.-C. Carrillo, C. R. De Galarreta, A. Baldycheva, H. Hayat, N. Youngblood, H. Bhaskaran, W. H. Pernice, and C. D. Wright, "Plasmonically-enhanced all-optical integrated phase-change memory," Optics express 27, 24724-24737 (2019)

${ }^{27}$ T. Domínguez Bucio, A. Z. Khokhar, G. Z. Mashanovich, and F. Y Gardes, "N-rich silicon nitride angled MMI for coarse wavelength division (de)multiplexing in the O-band," Optics Letters 43, 1251 (2018).

${ }^{28} \mathrm{~J}$. Hegedüs and S. R. Elliott, "Microscopic origin of the fast crystallization ability of Ge-Sb-Te phase-change memorymaterials," Nature Materials 7 399-405 (2008)

${ }^{29} \mathrm{~T}$. Matsunaga and N. Yamada, "A study of highly symmetrical crystal structures, commonly seen in high-speed phase-change materials, using syn- chrotron radiation," Japanese Journal of Applied Physics, Part 1: Regular Papers and Short Notes and Review Papers 41, 1674-1678 (2002).

${ }^{30}$ B.-S. Lee, J. R. Abelson, S. G. Bishop, D.-H. Kang, B.-k. Cheong, and K.B. Kim, "Investigation of the optical and electronic properties of ge2sb2te5 phase change material in its amorphous, cubic, and hexagonal phases," Journal of Applied Physics 97, 093509 (2005)

${ }^{31}$ T. Tsafack, E. Piccinini, B.-S. Lee, E. Pop, and M. Rudan, "Electronic, optical and thermal properties of the hexagonal and rocksalt-like ge2sb2te5 chalcogenide from first-principle calculations," Journal of Applied Physics 110, 063716 (2011).

${ }^{32}$ I. Friedrich, V. Weidenhof, W. Njoroge, P. Franz, and M. Wuttig, "Structural transformations of Ge2Sb2Te5 films studied by electrical resistance measurements," Journal of Applied Physics 87, 4130-4134 (2000).

${ }^{33}$ X. Liu, X. Li, L. Zhang, Y. Cheng, Z. Yan, M. Xu, X. Han, S. Zhang, Z. Zhang, and E. Ma, "New structural picture of the ge2sb2te5 phasechange alloy," Physical review letters 106, 025501 (2011).

${ }^{34}$ B. Chen, G. H. ten Brink, G. Palasantzas, and B. J. Kooi, "Crystallization kinetics of gesbte phase-change nanoparticles resolved by ultrafast calorimetry," The Journal of Physical Chemistry C 121, 8569-8578 (2017).

${ }^{35}$ W. De Cort, J. Beeckman, T. Claes, K. Neyts, and R. Baets, "Wide tuning of silicon-on-insulator ring resonators with a liquid crystal cladding," Optics letters 36, 3876-3878 (2011)

${ }^{36}$ A. Farmani, "Graphene plasmonic: Switching applications," Handbook of Graphene: Physics, Chemistry, and Biology , 455 (2019).

${ }^{37}$ L. A. Shiramin and D. Van Thourhout, "Graphene modulators and switches integrated on silicon and silicon nitride waveguide," IEEE Journal of Selected Topics in Quantum Electronics 23, 94-100 (2016).

${ }^{38}$ F. Gardes, G. Reed, N. Emerson, and C. Png, "A sub-micron depletion-type photonic modulator in silicon on insulator," Optics Express 13, 8845-8854 (2005)

${ }^{39}$ R. B. Priti, Y. Xiong, and O. Liboiron-Ladouceur, "Efficiency improvement of an o-band soi-mzi thermo-optic matrix switch," in 2016 IEEE Photonics Conference (IPC) (IEEE, 2016) pp. 823-824.

${ }^{40}$ N. A. Mohammed, H. S. A. Elnasr, and M. H. Aly, "Performance evaluation and enhancement of $2 \times 2$ ti: Linbo3 mach zehnder interferometer switch at $1.3 \mu \mathrm{m}$ and $1.55 \mu \mathrm{m}$," The Open Electrical \& Electronic Engineering Journal 6 (2012)

${ }^{41}$ M. Zhang, K. Chen, W. Jin, and K. S. Chiang, "Electro-optic mode switch based on lithium-niobate mach-zehnder interferometer," Applied optics 55, $4418-4422$ (2016)

${ }^{42}$ R. Amin, J. B. Khurgin, and V. J. Sorger, "Waveguide-based electroabsorption modulator performance: comparative analysis," Optics express 26, 15445-15470 (2018)

${ }^{43}$ A. Joushaghani, J. Jeong, S. Paradis, D. Alain, J. S. Aitchison, and J. K. Poon, "Wavelength-size hybrid si-vo 2 waveguide electroabsorption optical switches and photodetectors," Optics express 23, 3657-3668 (2015).

${ }^{44}$ N. Dupuis, A. V. Rylyakov, C. L. Schow, D. M. Kuchta, C. W. Baks, J. S. Orcutt, D. M. Gill, W. M. Green, and B. G. Lee, "Ultralow crosstalk nanosecond-scale nested $2 \times 2$ mach-zehnder silicon photonic switch," Optics letters 41, 3002-3005 (2016)

${ }^{45}$ A. M. Al-Hetar, A. B. Mohammad, A. S. M. Supa'At, and Z. A. Shamsan, "Mmi-mzi polymer thermo-optic switch with a high refractive index contrast," Journal of Lightwave Technology 29, 171-178 (2010).

${ }^{46} \mathrm{R}$. B. Priti and O. Liboiron-Ladouceur, "A broadband rearrangable nonblocking mzi-based thermo-optic o-band switch in silicon-on-insulator," in Advanced Photonics 2017 (IPR, NOMA, Sensors, Networks, SPPCom, PS) (Optical Society of America, 2017) p. PM4D.2.

${ }^{47}$ J. V. Campenhout, W. M. J. Green, S. Assefa, and Y. A. Vlasov, "Lowpower, $2 \times 2$ silicon electro-optic switch with 110-nm bandwidth for broadband reconfigurable optical networks," Opt. Express 17, 24020-24029 (2009).

${ }^{48}$ L. Lu, S. Zhao, L. Zhou, D. Li, Z. Li, M. Wang, X. Li, and J. Chen, "16x16 non-blocking silicon optical switch based on electro-optic mach-zehnder interferometers," Opt. Express 24, 9295-9307 (2016).

${ }^{49}$ L. Qiao, W. Tang, and T. Chu, "Ultra-large-scale silicon optical switches," 2016 IEEE 13th International Conference on Group IV Photonics (GFP) 1-2 (2016).

${ }^{50} \mathrm{~L}$. Qiao, W. Tang, and T. Chu, "32x32 silicon electro-optic switch with built-in monitors and balanced-status units," Scientific Reports 7, 42306 (2017). 


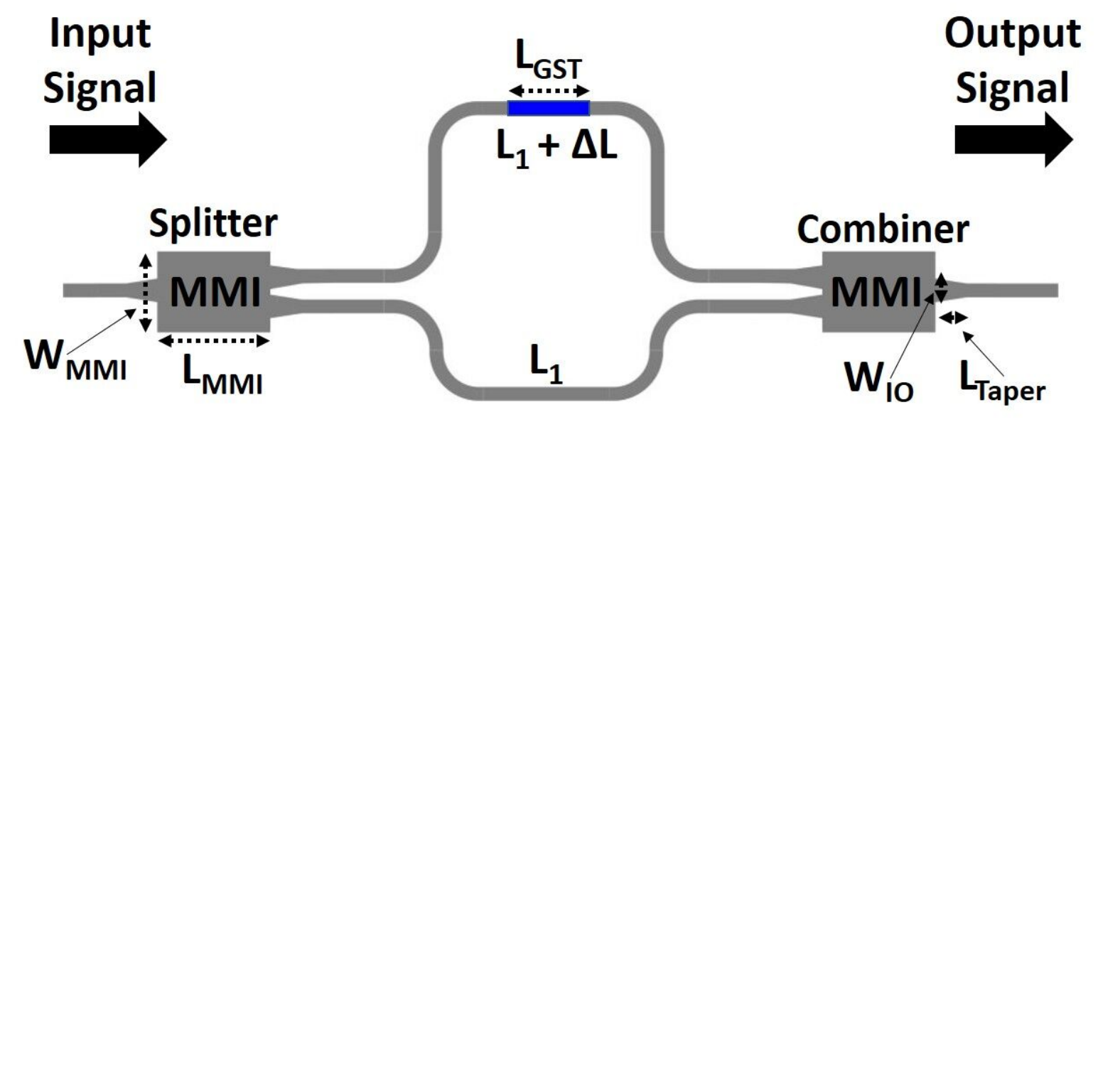


(a)
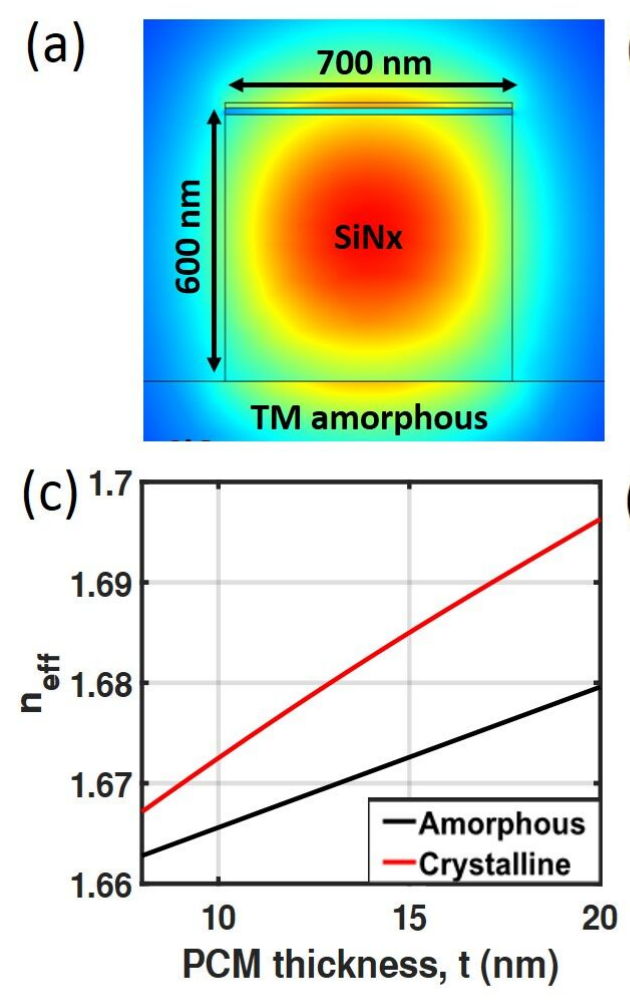

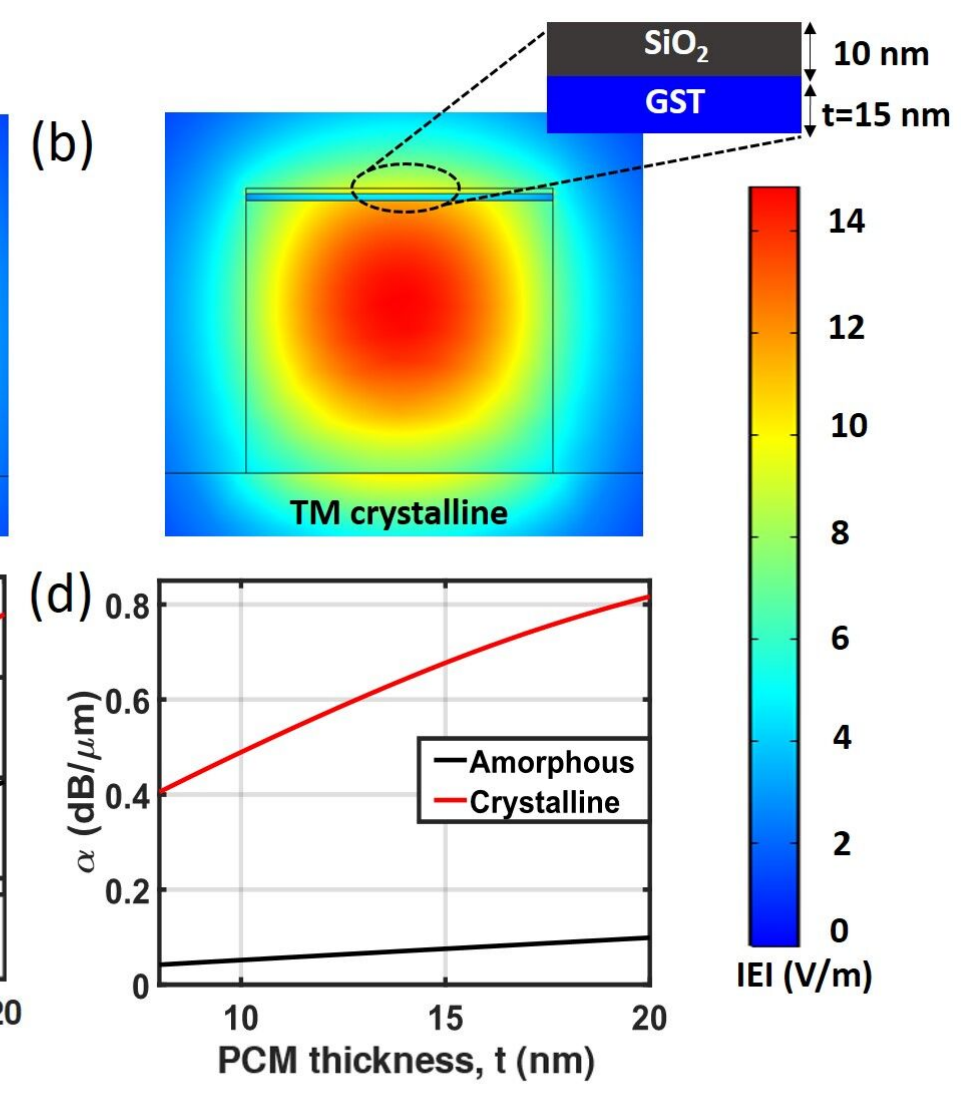




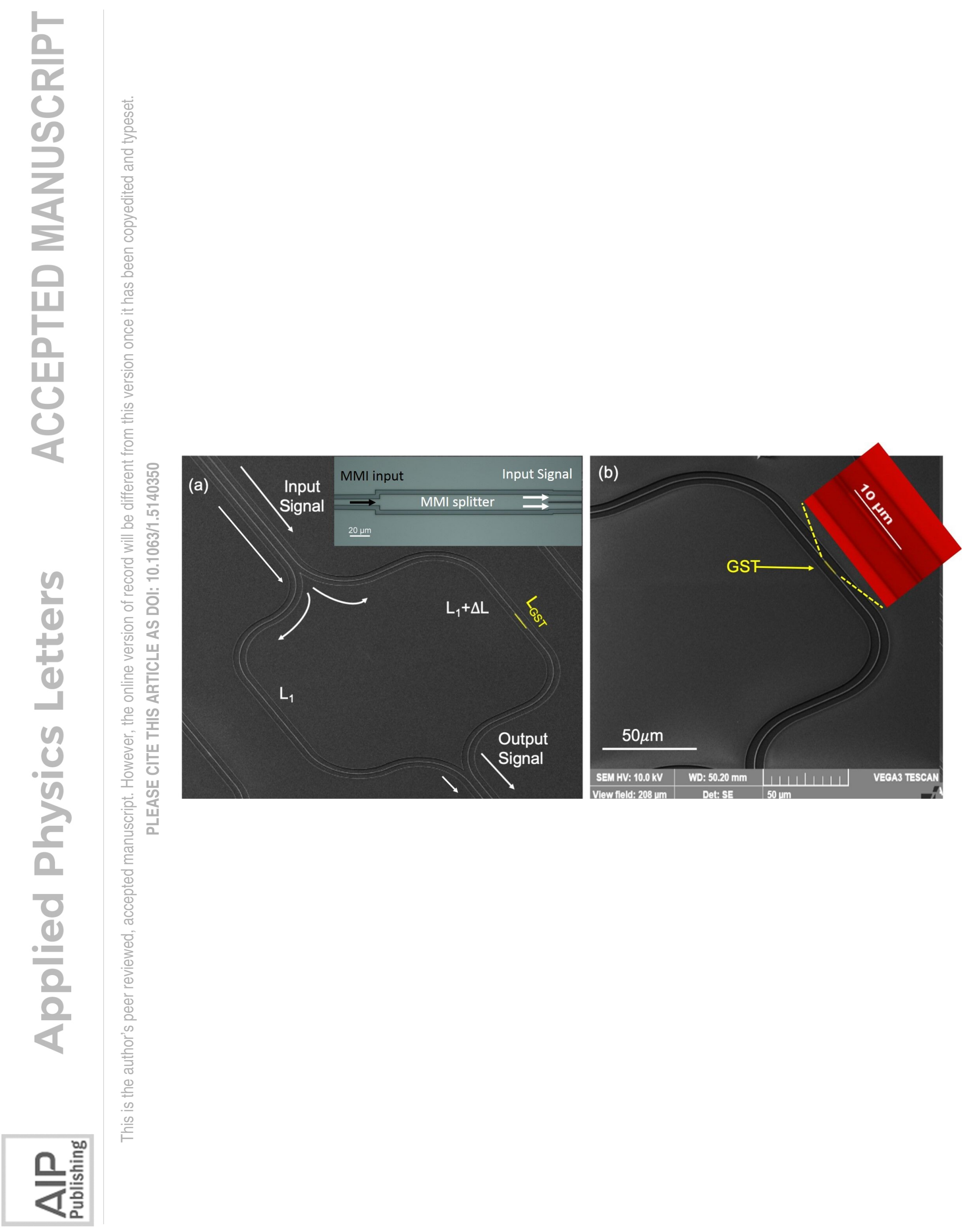




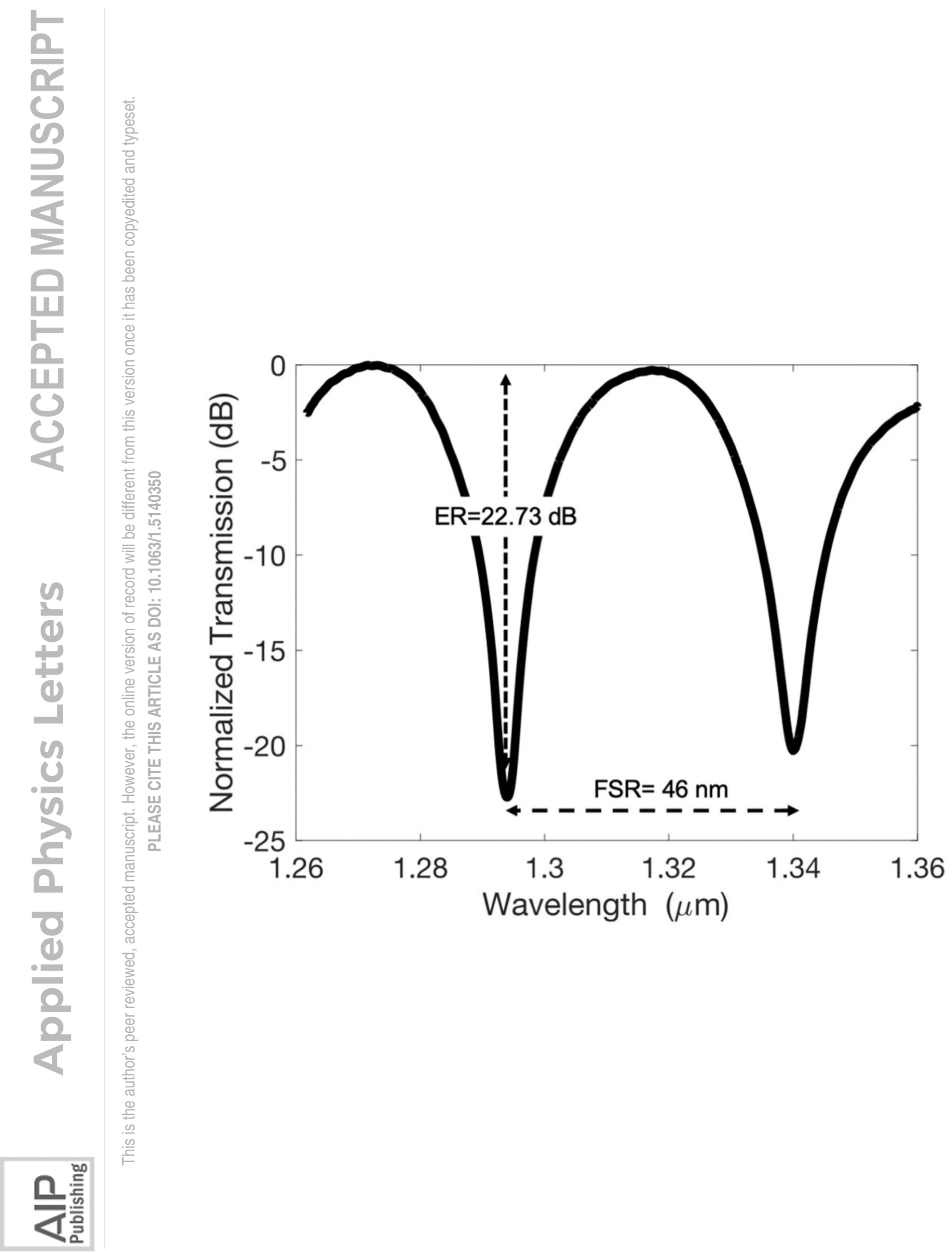




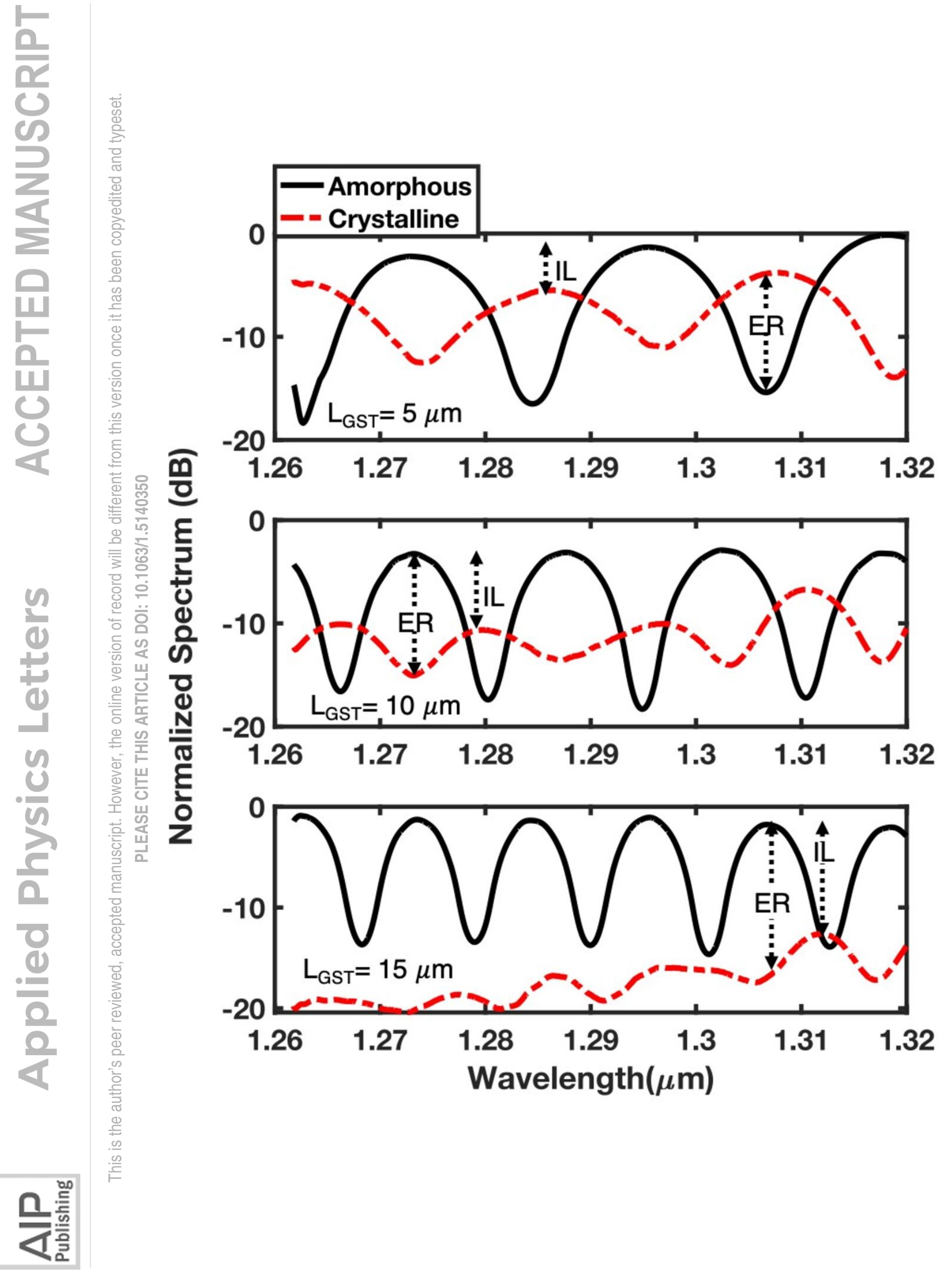




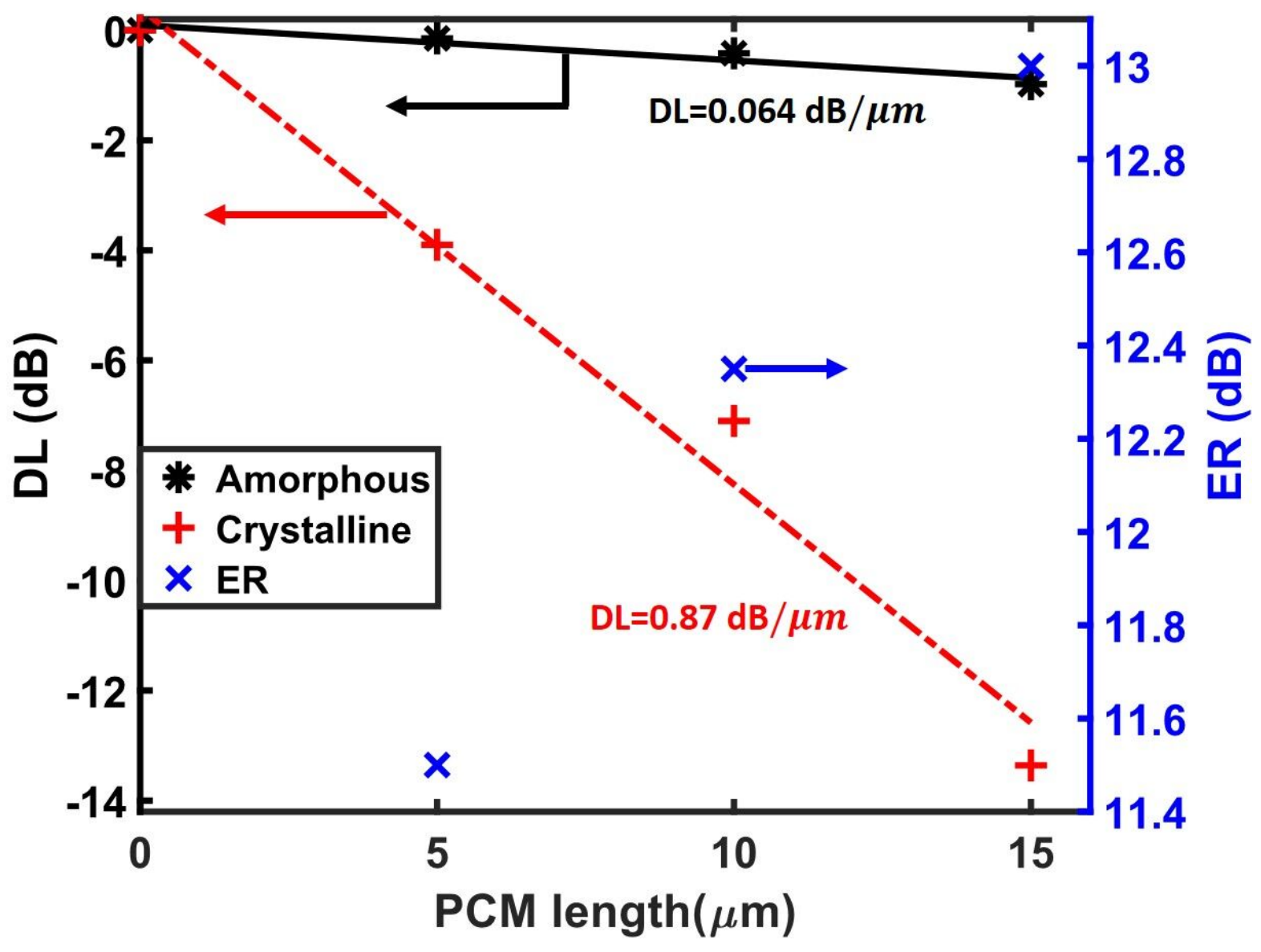

